The Journal of Public Space

2016 | Vol. I, n. I

\title{
VIEWPOINT
}

\section{Magic Carpet. \\ Re-envisioning Community Space in Hong Kong}

Hendrik Tieben

The Chinese University of Hong Kong, Hong Kong

School of Architecture

hktieben@cuhk.edu.hk

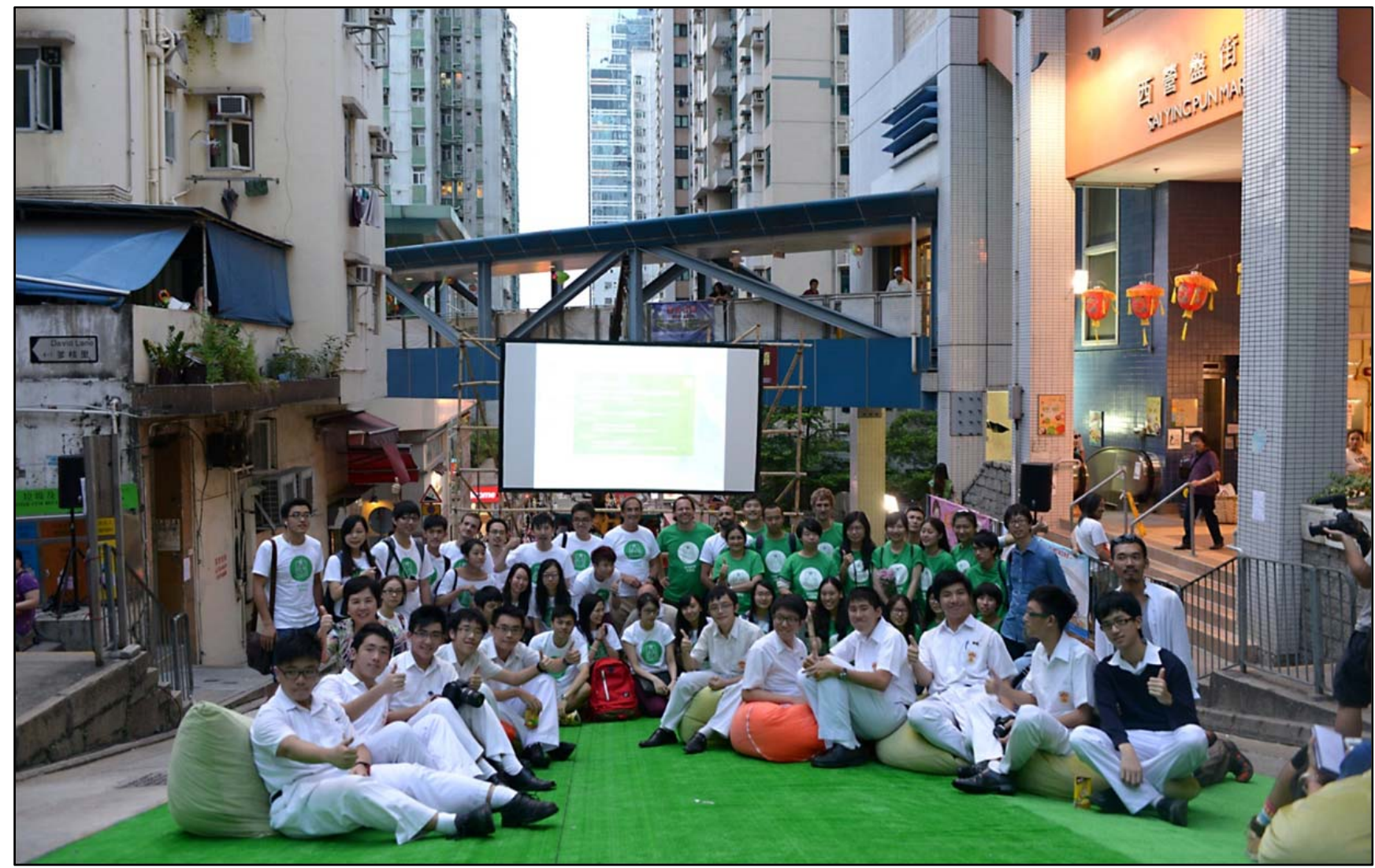

The Magic Carpet project team in Centre Street, Sai Ying Pun, Hong Kong, just few hours before the moonlight cinema event, Mid-Autumn Festival, 2013.

Magic Carpet was launched in 2013 by Hendrik Tieben of the School of Architecture and Anthony Fung of the School of Journalism and Communication at The Chinese University of Hong Kong, as an outreach program combining documentation of daily life, community engagement and urban design. The project was first held in Sai Ying Pun in 2013 and was subsequently brought to Tin Shui Wai (20I4) and To Kwa Wan (20I6).

Magic Carpet transforms a public space into an outdoor cinema in which movies about the neighbourhood are shown. The movies are produced by local secondary school 
students, following a series of workshops that prepare them to conduct video-interviews with the community.

At Magic Carpet, community members and the general public interact with each other, building a stronger bond between themselves while empowering them to re-envision the possibilities of public space together.
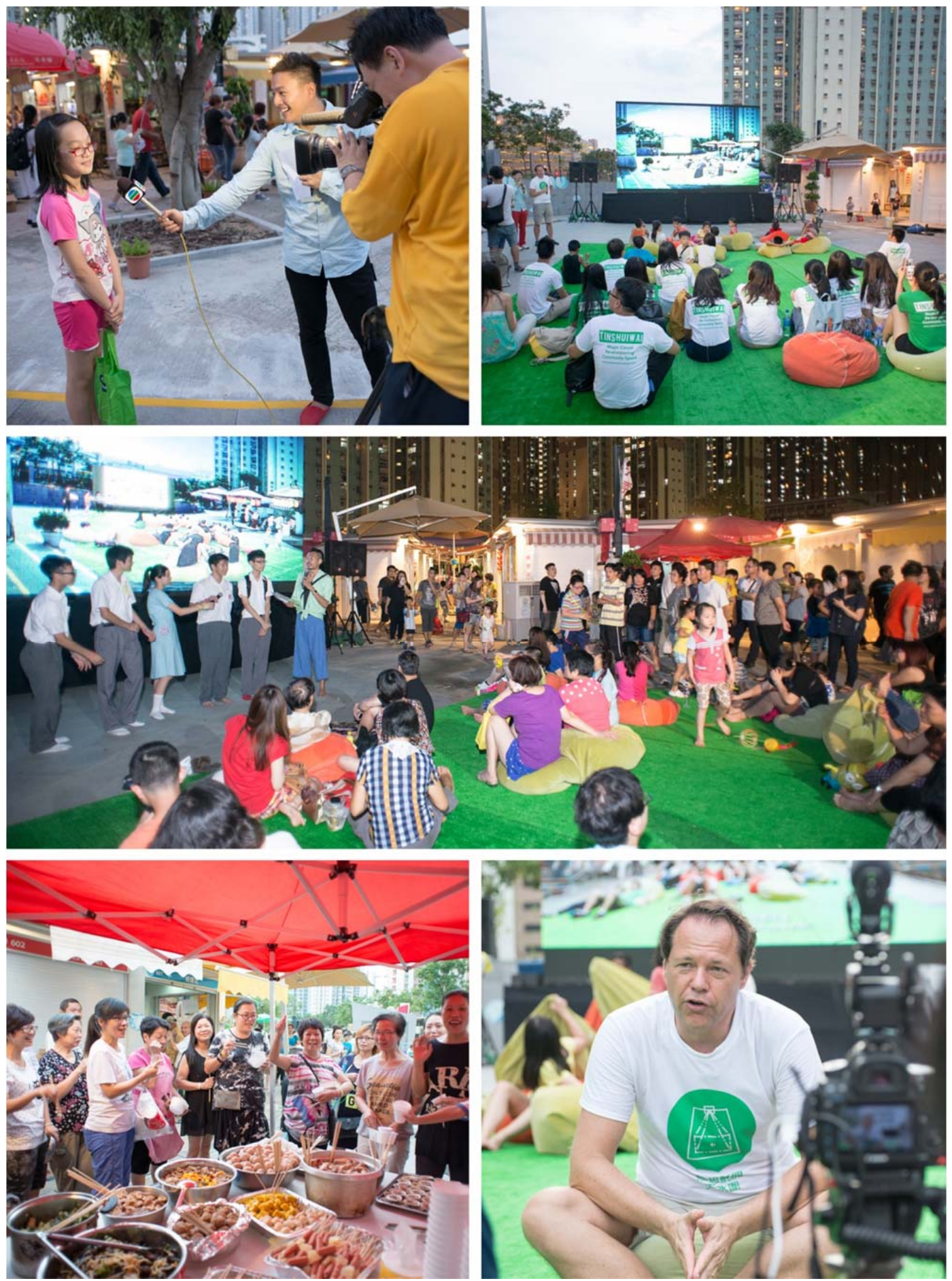

Pictures from the Magic Carpet event held in Tin Shui Wai in 2014.

I52 | The Journal of Public Space, I(I), 2016 | ISSN 2206-9658

(C) Queensland University of Technology 

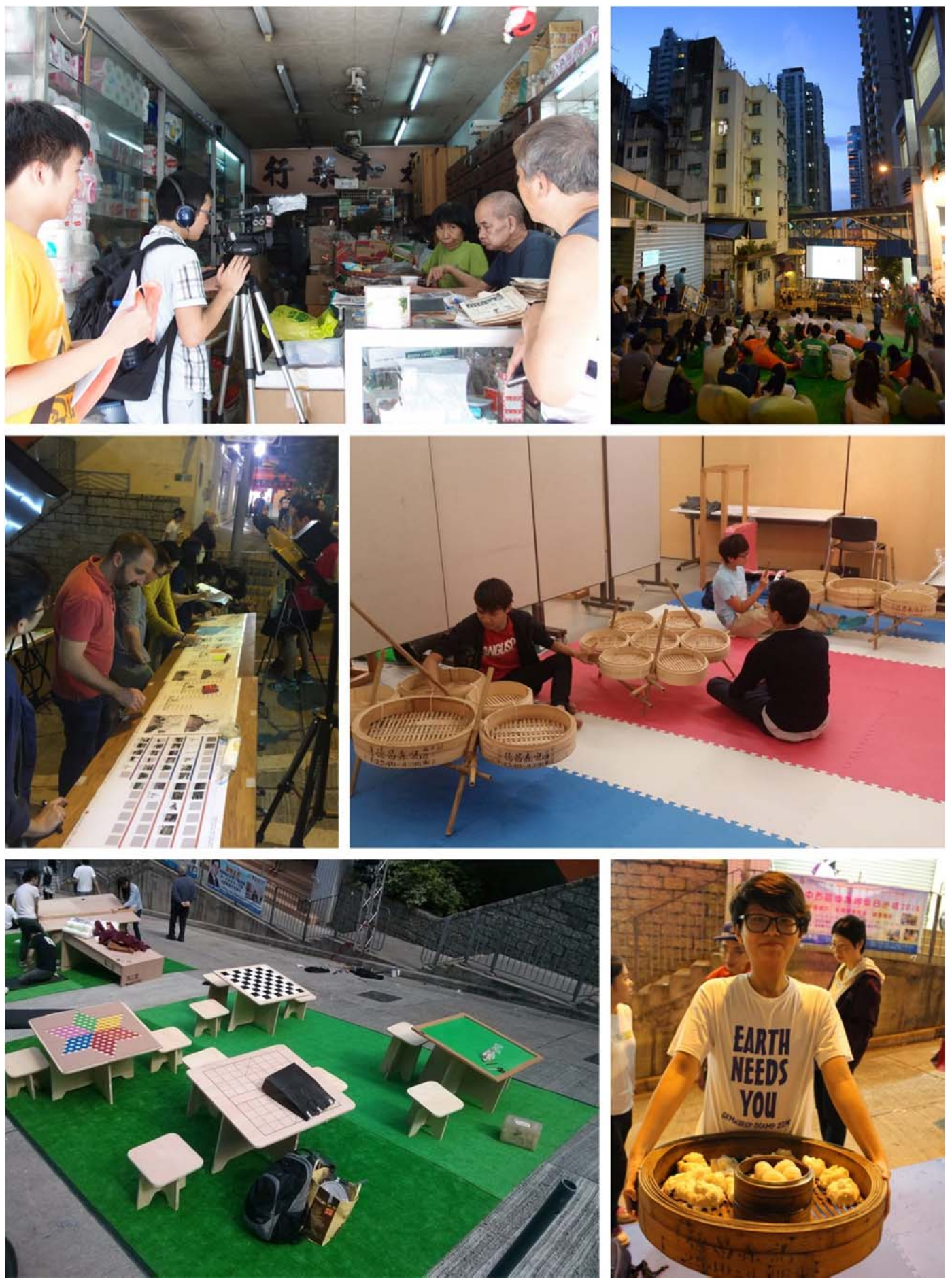

Pictures from Magic Tables, a design-build project + public event focusing on the making of community space. CUHK urban studies students collaborated with residents of different streets to engage in a friendly competition to "build" tables that allow stronger social interaction in the district.

Magic Carpet is one of a series of projects carried out by the same team to transform public space. A further initiatives has been Magic Tables - Remaking Community Space in Sai Ying Pun (20I5), in which Urban Studies students from CUHK built street furniture 
for social gathering. The latest Magic Carpet project focuses on the district To Kwa Wan, which is known for its grass-roots and migrant population and is currently transforming rapidly due to the construction of a new metro-line.

The Magic Carpet project has been supported by the Knowledge Transfer Fund of The Chinese University of Hong Kong and the Quality Education Fund, Hong Kong. Each project has been linked to studios of CUHK to engage urban studies and design students in community projects.

A parallel Magic Carpet project is organized by the National Taiwan University in Taipei. Results of both Magic Carpet projects will be discussed on a forthcoming book publication in late 2016.

\section{Credits}

Project initiator:

Hendrik Tieben, Associate Professor, School of Architecture

The Chinese University of Hong Kong

Project partners:

- Kwang Hwa Information and Culture Centre

- Conservancy Association Centre for Heritage

- Sai Ying Pun residents

Project team at CUHK:

- William Sin

- Janice Leung

- Francesco Rossini

- Yip Kai Chun

- Students of the BSSc in Urban Studies Program

Sponsor:

- Kwang Hwa Information and Culture Centre

Food sponsors:

- Pacific Island Hotel

- Sai Ying Pun Community

www.magiccarpet.hk

\section{To cite this article:}

Tieben, H. (20I6). Magic Carpet: Re-envisioning Community Space in Hong Kong. The Journal of Public Space, I(I), I5I-I54, DOI: 10.5204/jps.vlil.I7

This article has been accepted for publication in The Journal of Public Space. Please see the Editorial Policies under the 'About' section of the journal website for further information.

\section{(c) $\left(\begin{array}{l}\text { () } \\ \mathrm{BY}\end{array}\right.$}

This work is licensed under a Creative Commons Attribution - Non Commercial 4.0 International License. https://creativecommons.org/licenses/by-nc/4.0/ 